Cronfa - Swansea University Open Access Repository

This is an author produced version of a paper published in :

Breastfeeding Medicine

Cronfa URL for this paper:

http://cronfa.swan.ac.uk/Record/cronfa14800

\title{
Paper:
}

Dowling, S. \& Brown, A. (2013). An Exploration of the Experiences of Mothers Who Breastfeed Long-Term: What Are the Issues and Why Does It Matter?. Breastfeeding Medicine, 8(1)-52.

http://dx.doi.org/10.1089/bfm.2012.0057

This article is brought to you by Swansea University. Any person downloading material is agreeing to abide by the terms of the repository licence. Authors are personally responsible for adhering to publisher restrictions or conditions. When uploading content they are required to comply with their publisher agreement and the SHERPA RoMEO database to judge whether or not it is copyright safe to add this version of the paper to this repository. http://www.swansea.ac.uk/iss/researchsupport/cronfa-support/ 


\section{An Exploration of the Experiences of Mothers Who Breastfeed Long-Term: What Are the Issues and Why Does It Matter?}

Article in Breastfeeding Medicine · November 2012

Impact Factor: 1.25 · DOI: 10.1089/bfm.2012.0057 · Source: PubMed

CITATIONS

6

2 authors:

\section{Sally Dowling}

University of the West of England, Bristo 6 PUBLICATIONS 31 CITATIONS

SEE PROFILE
Amy Brown

Swansea University

57 PUBLICATIONS 452 CITATIONS

SEE PROFILE 
This is a post print of the article

Dowling, S., \& Brown, A. (2013). An exploration of the experiences of mothers who breastfeed long-term: What are the issues and why does it matter? Breastfeeding Medicine, 8(1), 45-52.

It can be found here

http://online.liebertpub.com/doi/abs/10.1089/bfm.2012.0057 


\title{
An exploration of the experiences of mothers who breastfeed long-term: what are the
} issues and why does it matter?

\author{
Sally Dowling, MPH, MFPH, MA, BA, RMN ${ }^{1}$ \\ Bristol and Amy Brown, $\mathrm{PhD}, \mathrm{MSc}, \mathrm{BSc}^{2}$ \\ ${ }^{1}$ University of the West of England, \\ ${ }^{2}$ Swansea University
}

\section{Corresponding Author}

Dr. Amy Brown

College of Human and Health Sciences

Swansea University, UK

SA2 8PP

+441792518672

Email: a.e.brown@swansea.ac.uk

Dowling's PhD research (on which elements of this paper are based) was supported by a studentship from the Faculty of Health and Life Sciences, University of the West of England, Bristol, 2007-2010.

No funding was received by Brown for this work.

The authors declare no conflicts of interest with respect to the authorship and/or publication of this article. 


\begin{abstract}
$\underline{\text { Abstract }}$
Background: The World Health Organisation (WHO) promotes breastfeeding for two years and beyond. Despite this, only $25 \%$ of women in the UK are breastfeeding at all by six months postpartum with a minority of women breastfeeding beyond the first year. Those who do often report feeling ridiculed or alienated in their choice. Here, the aim was to examine the experiences of women who chose to breastfeed longer-term and to seek insight into the ways they felt breastfeeding could be normalised past infancy.
\end{abstract}

Methods: The study combined two qualitative data sets which explored maternal attitudes and experiences towards longer-term breastfeeding. In study one a combination of methods were used, including interviews, to explore the challenges, attitudes and experiences faced by women who had breastfed for over six months postpartum. In study two, 1319 mothers who were pregnant of had an infant aged $0-2$ years completed an open ended questionnaire exploring their attitudes towards longer-term breastfeeding.

Results: Mothers who had experience of longer-term breastfeeding described how they faced negative attitudes and criticism from others including the perceptions that longer-term breastfeeding was comical, bizarre and pointless. Mothers discussed ways in which longerterm breastfeeding could be normalised rather than promoted, targeting health professionals and society instead of encouraging mothers themselves. Key ideas included greater visual representation, increasing knowledge and removing stigma.

Conclusions: Longer-term breastfeeding needs to be normalised to increase acceptance and in turn reduce the negative attitudes that mothers often face despite following WHO guidance. Greater support is needed from Health Professionals and Health Policy. 


\section{Introduction}

Breastfeeding is widely acknowledged to be beneficial for both maternal and infant health ${ }^{1,2}$. Consequently, the World Health Organisation recommends that babies are exclusively breastfed for six months postpartum and that following this they continue to receive their mother's milk for 'up to two years or beyond' alongside complimentary foods ${ }^{3}$. These international targets are broadly supported at a national level in many countries through government policy and public health promotion activities. The UK Department of Health for example echoes this guidance although talks about less precisely about duration of breastfeeding and how 'can be continued' alongside solid food ${ }^{4}$.

However, in the UK, although breastfeeding initiation rates are good, many mothers stop breastfeeding within the first few days and weeks with only twenty five per cent of mothers still breastfeeding at six months, less than one per cent exclusively ${ }^{5}$. The consequence of this is that breastfeeding promotion, literature and practical support often focus on increasing initiation rates and supporting mothers during the first few weeks postpartum ${ }^{6}$. Little research considers the experiences of, or factors influencing, maternal decision to breastfeed past six months or indeed considers the implications for breastfeeding policy and practice.

The literature exploring the health and other benefits of breastfeeding is substantial but often only focuses on breastfeeding in the early days and weeks ${ }^{7,8,9}$. However, a handful of studies do explore the positive effects of longer-term breastfeeding upon both infant and maternal health $^{10,11}$. Reference is also often made by those who are interested in longer-term breastfeeding to the work of anthropologist Dettwyler - who suggested a 'hominid blueprint' for weaning, with the 'natural' age of ceasing breastfeeding ranging from two and a half to seven years ${ }^{12}$. 
The incidence of long-term breastfeeding in the UK is estimated to be fairly low, although official measurement can be difficult as even the UK quinquennial Infant Feeding Survey only records breastfeeding up to nine months postpartum. Figures collected in 2005 showed that only $18 \%$ of women were breastfeeding at all at this time ${ }^{13}$. With numbers of mothers who artificially feed from birth or who are exclusively artificially feeding by six weeks postpartum far outweighing this small percentage, longer-term breastfeeding is thus not a common, normal or expected behaviour in the UK. Indeed, the UK has been described as a 'formula feeding culture' with the biological norm of long-term breastfeeding at odds with the social and cultural norm ${ }^{14}$. Breastfeeding is often associated with very young infants, and considered unnecessary for older babies and children. Women who breastfeed 'long-term' therefore find themselves in a difficult position as they follow public health guidance whilst at the same time engaging in behaviour generally considered by others to be bizarre, even unhealthy ${ }^{15,16}$.

The aim of this paper was to consider women's experiences and perceptions of long-term breastfeeding alongside examining what would make women who choose to follow Public Health guidance feel more supported and accepted in their choice. Within this it explores the role of Health Practitioners and contributes to the wider debate of how to increase the acceptance, normality and incidence of breastfeeding as a whole in the UK

\section{Methods}

This paper brings together the findings of two separate but complementary studies exploring women's experiences and perceptions of longer- term breastfeeding. As the aims of both studies were so similar, the decision was made to combine and consider the factors in one empirical paper to add greater depth, participant numbers and perspectives. Study One 
(Dowling) used a combination of ethnographic methods (face-to-face and online interviews with small numbers of women and participant observation in breastfeeding support groups) whereas Study Two (Brown) utilised an open ended questionnaire with a larger group of breastfeeding women.

\section{Participants}

Ethical approval was granted for each study through the relevant University Research Ethics Committees. All applicable institutional and governmental regulations concerning the ethical use of human volunteers were followed during the conduct of each research project.

In Study One, ten women who had breastfed for over six months postpartum were interviewed either face-to-face $(n=6)$ or by using online asynchronous interviews using email $(\mathrm{n}=4)^{17}$. In addition contact was made with over eighty breastfeeding women via participant observation in breastfeeding support groups. Due to low numbers of women choosing to breastfeed past six months postpartum, purposive and snowball sampling was used to recruit participants. Data collection took place over a period of eighteen months during the $2008-$ 2010 period. The work was informed by feminist beliefs in the conduct and interpretation of research and was carried out from an 'insider' perspective ${ }^{18}$ as the researcher was also breastfeeding throughout project design, data collection and analysis ${ }^{19}$.

In Study Two 1319 participants completed an open ended questionnaire exploring their experiences of breast and formula feeding and attitudes towards breastfeeding promotion. Participants were either currently pregnant or had given birth in the last two years and were initially recruited from local mother and baby groups and through online parenting forums based in the UK. It was not a requirement for participants to have experience of longer-term breastfeeding although a proportion of the sample did. Snowball sampling through word of mouth led to the questionnaire being distributed by mother and baby groups across the UK 
and through further online adverts in the form of online blogs, Facebook and twitter. Data collection took place over a three month period during 2011.

\section{Measures}

In both studies interview questions explored how the participants' breastfeeding was perceived both by themselves (before and after they had breastfed) and how they felt others perceived breastfeeding (once they were breastfeeding long-term).

Study One was also concerned with how women experienced living as long-term breastfeeders and in the sources of support that enabled them to continue breastfeeding when most other women had stopped. Interviews were open-ended and unstructured although a topic guide was used to focus discussion. Participant observation concentrated on what it was like to be in environments supportive of breastfeeding as well as how breastfeeding longer-term was discussed amongst participants.

Study two used an open ended online questionnaire to examine attitudes towards breastfeeding promotion. Within this, three specific questions targeted beliefs and experiences of longer - term breastfeeding and promotion (1) 'If you breastfed past six months what were your experiences of doing so?', (2) 'Did you receive any information or promotion of breastfeeding past the age of six months? What?' and 'Should breastfeeding promotion target breastfeeding older babies and toddlers? How?'. Participants were encouraged to describe their experiences rather than giving closed responses and indeed did so, giving detailed qualitative responses.

\section{Data Analysis}

The two data sets were considered separately but similar data analysis procedures were carried out for both. All data was anonymised and imported into NVivo for thematic analysis. 
A simple thematic approach was used to generate qualitative description ${ }^{20,21}$; each script was read through to identify emerging themes which were then grouped into key themes and sub categories. In Study Two these were confirmed by two independent coders with agreement found in over $90 \%$ of cases. In Study One all coding was undertaken by the researcher and discussed during doctoral supervision.

Data saturation principles were followed during the interviews with data collection continuing until it was felt that no new themes or ideas were emerging. In Study Two the sample size exceeded recommended minimums ${ }^{22,23}$. Quantitative data collection was allowed to run for a set period of time with numbers far exceeding expectations with data saturation clearly found.

\section{$\underline{\text { Results }}$}

In Study One ten women were interviewed in total; at the time of the interviews they had breastfed for between four months (although had experience of breastfeeding for longer) and six and a half years. Inclusion was predicated on having all having breastfed for at least six months, within the previous five years. Women interviewed online had higher educational qualifications and were more likely to be working outside the home than women interviewed face-to-face. In addition contact was made with over eighty women in three breastfeeding support groups; these women ranged in age from early twenties to mid-forties and were breastfeeding babies and children from new-borns to four years old. Participant observation allowed for increased understanding of the support gained by breastfeeding women as well as contact with women from a greater range of social and economic circumstances. In addition the use of three methods of data collection enabled triangulation between methods, increasing the 'trustworthiness' of the final data $\operatorname{set}^{24}$. 
In study two mean age of the participants was 30.69 (SD: 4.52) with a mean years in education of 14.01 (SD: 3.60). 394 participants were currently pregnant [189 with their first infant] and 1145 parents had an infant age $0-2$ years. Mean age of infant was 39 weeks [SD: 37.25]. 220 participants had both an infant within the age range and were also pregnant. A range of breast and bottle feeding experiences were seen. $45.2 \%$ of mothers were breastfeeding at the time of the questionnaire. Of those who had initiated but stopped breastfeeding, mean duration of breastfeeding amongst the sample was 18.2 weeks with a range from 1 day to 2 years. $32.4 \%$ of the sample had an extended breastfeeding duration of over six months.

Overall, a variety of themes were produced describing mothers beliefs of how long term breastfeeding is portrayed, experiences of doing so and how supported they felt in their choices. Suggestions for how health professionals could provide wider support and understanding of the issues mothers face when long term breastfeeding are considered. Notably, a key premise running throughout the responses and across thematic categories was the idea that the mothers themselves had often thought differently of long term breastfeeding before they had breastfed an infant themselves. The importance of this change, the recognition of why these reactions had occurred and how this could be used to influence social and cultural norms is discussed.

\section{What are mothers experiences of long term breastfeeding?}

\section{Breastfeeding is synonymous with young babies}

A central theme found throughout the responses was the idea that breastfeeding is perceived to be for younger infants only. Mothers themselves noted that before they had given birth they had only visualised themselves breastfeeding a tiny infant, with little thought to how long they might continue. 
'You get it in your head it's going to be a little baby'

'Before my first child I would never have thought I would have fed him until he was nearly four'

Related to this many mothers commented that the recommendation to breastfeed exclusively for six months has become generally interpreted as 'breastfeed for six months and then stop'. Breastfeeding is perceived as something that happens up to six months of age followed by the promotion of 'follow-on milks' and the switch from breast to formula milk in the latter part of the first year. Many women see this as normal or inevitable which is problematic in terms of normalising the continuation of breastfeeding.

'I just assumed that there must be a time that everyone stops, that, you know, you...that's it and then that's that... but I never knew what that age was, I suppose either six months or twelve months, I didn't really know'

'My NCT teacher asked us how long we were supposed to breastfeed for ... cue lots of 'um 6 months' ...her answer: As long as you can'

Although once mothers were practising longer-term breastfeeding it became normal behaviour to them, many recounted how they had themselves had negative reactions to witnessing older infants' breastfeeding in the past. Mothers talked about being shocked or surprised when they first saw an older baby or child breastfeeding. Most recognised that this was because it was something unfamiliar, outside their experience and not a course of action that they had considered for themselves:

'I was like - 'look how big that child is!' it just seems weird, having this child that's walking around and just like comes on over, it's like ohhhhhh. You know, kind of just takes up your whole lap and demands some milk. Weird.... and then, 
and maybe shocking because...I never thought about feeding them that...you know, it just never even occurred to me'

\section{Longer-term breastfeeding is 'pointless'}

Mothers also described how those around them made the implicit assumption that once their baby had grown older they would no longer be breastfeeding despite the WHO recommendations to consider doing so until two years and beyond. The comments made to women about the length of time they breastfeed for indicate that, in many people's minds there is clearly a cut-off beyond which it is no longer acceptable (or even comprehensible) to 'still' be breastfeeding.

'about a year, when everyone else was doing something else and it was "Are you still feeding him? Are you still feeding him?" Now he's eighteen months they just assume that I've stopped, nobody's mentioned it for a while'

Within this it was common for the suggestion to be made that mothers were only continuing to breastfeed at this stage for their own needs or enjoyment.

'That was the hardest thing...going to stay with the in-laws and them thinking extended breastfeeding's very strange...they would say, you know, it's bizarre, it's completely unnatural, it's for you not him.'

At the most extreme some mothers were informed that by continuing to breastfeed they would harm their child.

'the psychologist actually told me that if I continue breastfeeding Janie to help her calm down that I am contributing to her developing an addiction later in life, to anything that she can take into her mouth - food, alcohol, cigarettes, or drugs!!!!' 
This reaction extended to Health Professionals. The majority of mothers reported that they had little support or guidance on longer - term breastfeeding, often being met by negative or crude attitudes.

'I got no information at all from health professionals ... the reassurance and education I got was from a breastfeeding older babies and beyond group on the Babycentre community website'.

'I had a really bad earache... and I'd gone back and forth to the doctor...she was prescribing something and I said 'can I take that when I'm breastfeeding?' and she said 'how long ago did you have your baby?' and he was about eighteen months old, and I'm like 'ages ago!'...And they expect him to be really little... So you do kind of think, what are people thinking, you know?'

\section{Longer term breastfeeding is bizarre or comical}

Mothers also described how they were often met with the reaction that what they were continuing to do was strange or abnormal behaviour. Others seemed confused as to why they would continue, labelling the mother as unnatural or deviant.

'people express surprise if you are breastfeeding longer than a year, like you are a funny person.'

'it's so important and it's so exciting and it's so easy and yet there's such misunderstanding around it...it's cloaked in all these bad connotations and words.'

Occasionally mothers also experienced ridicule or found themselves at the centre of jokes around their decision to breastfeed longer term. Breastfeeding a toddler was viewed as humorous but accompanied by disgust and unease. 
'My Health Visitor actually made crude and offensive jokes about 'still' feeding my toddler, even when he was barely a year old'.

'I got the bitty comment a lot ... and 'You'll still be feeding when he's at uni' even more'

This women refers here to the television comedy sketch ('Little Britain') in which an adult is seen breastfeeding (having 'bitty') in a range of situations. Critically, mothers often felt sad, angry or simply unsupported by those around them when these views were expressed even in jest.

'you're sort of out of synch with the common view aren't you? 'Cos I'm really proud that...I'm still feeding her, I think it's brilliant and it feels like totally the right thing to be doing. But it is sad, it's sad to be in a situation where you don't feel you can be proud of it because people don't understand'

As above, mothers themselves recognised that they too felt that longer-term breastfeeding was surprising or strange when they first encountered it. Emotions described were shock, surprise and discomfort even though these were women who then went on to breastfeed longterm (even though it was not something they had considered when the incidents they described took place).

'It was, it was surprising. It's, it's a funny visual...thing, the first time you see it, a, a big boy, with, you know, boots...long legs...I remember being, kind of, impressed, you know, thinking 'probably might not do that',

'mine wasn't so much when I first saw it as when I first heard about it and it was probably my conversation with Marie... she still fed her two and a half year old and I 
was a bit like, you know, 'how are you doing that? Or why are you doing that? And isn't that a bit weird?', you know, and, and she said 'well it just kind of happened'. And I remember thinking, 'this is not going to happen' ,

\section{Longer term breastfeeding is a negative experience for the mother}

A final theme raised was the presumption that breastfeeding was in some way a negative experience for the mother. The suggestion was commonly made that the mother had now done enough for her infant and should stop persevering. Alternatively mothers received comments suggesting that they were being masochistic or martyr like in their determination.

''oh well, you've done it for so long now you don't have to put yourself through it any more', and 'you've punished yourself enough by breastfeeding her' and 'if you don't give up now she's still going to be breastfeeding when she goes to school' and at the moment I've just been sort of smiling and nodding and not getting into it but then it's a problem, isn't it?'

'One told me I've 'gone beyond the call of duty' haven't I?

Overall, mothers faced many challenges predominantly related to the attitudes and behaviours of those around them when choosing to breastfed longer term. However mothers were adamant that longer - term breastfeeding was beneficial to their infants and should be encouraged. This leads naturally to the findings of study two which consider how the issues of longer - term breastfeeding can be overcome through challenging how breastfeeding is portrayed and promoted within the NHS. 


\section{Does longer term breastfeeding need to be promoted?}

In study two, participants were specifically asked whether they felt that longer - term breastfeeding should be promoted and encouraged by Health professionals and the NHS. Predominantly the response was positive; breastfeeding should not only be promoted in the early weeks and months but to be continued as long as the infant wishes to continue.

'Yes! People should be made aware of the benefits of breastfeeding past weaning and into toddlerhood (and the WHO recommendation that mothers breastfeed for two years and beyond). They should be told that this is normal, not weird, and that babies will still need lots of breast-milk once they start solids'.

Although, very few responses in the survey were against the concept of longer - term breastfeeding and believed that it should be encouraged and seen as the norm, approximately $25 \%$ of the sample did not believe that promotion or support for a longer duration was needed. This included respondents who had themselves breastfed for a longer period of time. Within this, some mothers however felt that resources and effort could be better targeted encouraging more women to initiate and continue breastfeeding in the early days.

'I think it's all about a good start and support at the beginning. If it doesn't work properly in the first few months people won't continue to breastfeed'.

'I feel that government and health agencies have enough of a job in persuading and encouraging mothers of small babies to breastfeed and should perhaps concentrate their efforts on this'.

Related to this was the concept that promotion at this stage was unnecessary. It was felt that once breastfeeding was established, mothers who find it relatively simple to continue and that 
many mothers who wanted to breastfeed until this stage were determined and confident enough to overcome the criticism that they face.

'I think targeting newborns is most effective as if the mother manages this she will probably naturally be more inclined to keep feeding when breastfeeding is established'.

A number of participants also made the important distinction that perhaps longer - term breastfeeding promotion needed to target the general public rather than the mothers themselves. They felt that mothers were generally motivated and able to continue breastfeeding if they wished but the greatest barrier they faced was the attitudes and behaviours of those around them. By equipping their family, peers and society as to how normal breastfeeding was, mothers would find it easier to continue.

'Perhaps with regard to educating the general public but I don't think I would find it that useful as a mother...what info would I need?'

Related to this, others suggested that promotion should target NHS professionals rather than the mothers themselves. Many mothers recalled how they received little information or guidance from health professionals with regard to breastfeeding once their infant was more than a few weeks old. Many faced attitudes that breastfeeding was simply not needed or found that professionals expected them to have stopped. It was common for participants simply not to be asked whether they were breastfeeding at any appointment.

'Train NHS staff to encourage feeding older children rather than the surprise / shock / negativity you currently get if you are feeding 'still' after 4 months' 
However, overall there was a strong feeling amongst both groups of participants that more needed to be done to highlight the importance of longer - term breastfeeding, encourage mothers to continue and support them in doing so.

\section{How could long term breastfeeding be normalised?}

The results from both studies showed that mothers experienced criticism, barriers and ridicule when continuing to breastfeed past a few months. The overwhelming attitude was that longer - term breastfeeding needed to be viewed as something 'normal' and acceptable rather than an unusual behaviour. Within their accounts of their experiences of longer-term breastfeeding, mothers suggested a number of ways in which longer term breastfeeding could be normalised and promoted in a healthy fashion. Suggestions including greater visual representation, increasing knowledge and removing stigma from the context of longer - term breastfeeding, although credence was given to the importance of a gradual change in attitudes to enable society to adapt.

The most central concept was that longer-term breastfeeding had to be promoted as a stronger social and cultural norm rather than abnormal behaviour. People had to start accepting that longer - term breastfeeding was not only the biological norm but an acceptable and common behaviour, seen as important in many cultures.

'I think it should normalise it more, just having a tiny newborn makes people think only tiny babies should be breastfed'

'people need to realise that breastfeeding a child of any age is perfectly normal'

Within this mothers felt that more needed to be done to increase knowledge as to why longer term breastfeeding is important for infants. Emphasis had to be placed on the knowledge that longer - term breastfeeding had health benefits over and above follow on milk or cow's milk, 
that milk was still an important element of an infant's diet and that breastfeeding offered many more additional benefits to the older infant.

'People should realise that babies are not biologically designed to be weaned from the breast at six months, a year or even two years'.

'Promotion could explore issues and myths around teeth, speech, biology, continued benefits physically and emotionally, feeding whilst pregnant and tandem feeding'.

Alongside this, challenging the misconceptions and taboos that are held in society regarding the concept of longer - term breastfeeding was felt to be critical.

'People are so bizarrely uncomfortable about children nursing after the age of a year or so. It seems really unhealthy the way many people are uncomfortable with it'

A key element to this was supporting longer- term breastfeeding to become more visually represented in public, so that seeing an older infant breastfeeding in public would be considered more socially acceptable rather than something 'shocking'. One popular suggestion was the idea that NHS and Government material promoting breastfeeding should include pictures of older children breastfeeding.

'Posters, TV adverts, hand outs from professionals'.

'Promotional materials should have a section indicating that it is ok to breastfeed for as long as possible with...pictures of older babies feeding rather than younger'

Related to this was the concept of moving away from the language used in promotion that highlighted the six months timeframe. More emphasis should be placed on breastfeeding for a year or longer with the idea that solid foods should be introduced alongside milk at around six months old. 
'...stop the promotion of "up to 6 months" which leads people to think that this is a maximum target'

'I know lots of people who happily breastfed for 6 months then felt they had done their bit and started formula feeding...there should be more information on the continued benefits.'

One suggestion on how this could work by removing the idea of a time frame was the concept that breastfeeding could continue for as long as the infant wishes to or the mother is happy to.

'The message needs to be breastfeed as long as you both want to, and add food at around 6 months...'

However, the importance of timing of introduction of materials both to the mother and society itself was raised. It was recognised that perhaps exposure needed to be gradual and integrated into promotional materials and publications a step at a time. The data clearly shows that even these mothers who themselves were long-term breastfeeding had felt shocked or surprised before their experiences when encountering older infants breastfeeding. On the one hand images of breastfed children needed to become more common place but this needed to be done in a sensitive way to ensure that a shock factor was not created.

'...I think if you promote toddler feeding to a pregnant or new mum too much it can be a bit terrifying as toddlers look huge and rather strange. Perhaps more information should be given in stages and gently filter in the idea of breastfeeding an older child?...'

Participants felt that perhaps although society gradually needed to become adjusted to the idea, and view, longer - term breastfeeding, perhaps new parents needed the information one 
step at a time. Initially information needed to target establishing breastfeeding but over time literature could emphasise continued breastfeeding. On this note, one challenge to promotion was the idea that encouraging mothers to continue breastfeeding for a longer period may lead to more mothers feeling under pressure if they cannot continue when they return to work or their infant decides to no longer feed. Mothers felt that emphasis needed to be placed on how well mothers had done to breastfeed at all, but continued breastfeeding was beneficial.

'I do think there needs to be a fine line - the idea of feeding a nearly three year old was terrifying when I had a new baby but its something that has developed with our relationship'.

'Start off with smaller chunks...things like 'if you breastfeed for two weeks....if you breastfeed for one month...and then build up'.

Overall, the key message that emerged from the data was that steps needed to be taken to normalise longer-term breastfeeding. In general mothers did not feel the approach needed to be promoted and publicised, but more that a gradual remodelling and conceptualisation of longer-term breastfeeding was needed to enable more mothers to feel accepted and comfortable in following Public Health guidance.

\section{$\underline{\text { Discussion }}$}

This paper presented the findings from two studies exploring the portrayal and experience of longer - term breastfeeding. Key themes focussed around the central standpoint that longer term breastfeeding, although the biological norm, was not considered a social norm. Indeed, longer - term breastfeeding was viewed as something abnormal, strange or harmful or indeed excluded from public portrayal at all through the absence of literature pertaining to discussion and photos of older infants breastfeeding. This echoed previous work in the area e.g. Rempel 
(2004) and Gribble $(2007,2008)$ where women felt in the minority, isolated in their choices and as if they were doing something abnormal ${ }^{25}, 26,27$.

The question arises as to how the perception of longer - term breastfeeding can be normalised. One theme that arose from the data was that mothers felt that longer - term breastfeeding did not necessarily need to be directly encouraged and promoted by health professionals (with effort perhaps being focussed on the earlier stages, with the expectation that if breastfeeding got off to a good start, more mothers would naturally continue). However, mothers, especially those who had experience of longer - term breastfeeding strongly wished for it to be seen as more natural, normal and acceptable and for society and others to feel comfortable with the concept. This brings the discussion back to how mothers believed this state could be gradually reached, targeting not only parents but health professionals and wider society too.

One criticism was that breastfeeding literature rarely extends to portraying an image of a young infant, presenting the assumption that breastfeeding is only for newborn infants. One suggestion could thus be that pictures are presented in NHS leaflets promoting breastfeeding or indeed general parenting such as the Birth to Five publication in the UK which covers all aspects of infancy and pre-school parenting ${ }^{28}$. Caution however perhaps needs to be given to inclusion of such pictures in newborn sections due to the potential 'shock' factor experienced even by mothers in the early stages of seeing an older child breastfeeding who themselves went on to long - term breastfed. Inclusion could naturally occur when discussing issues with older babies and toddlers.

The second and related issue could involve greater training and education in the benefits of breastfeeding older infants and children for health professionals working with mothers. A common finding was that mothers who longer - term breastfed often encountered negative 
attitudes or simply a lack of awareness or understanding from doctors and health visitors, despite having WHO recommendations on their side.

Finally, the normalisation of longer - term breastfeeding needs to be targeted at a society wide level. This is a difficult conundrum to manage, as mothers of younger infants often report disdainful attitudes and ignorance from family members, peers and the general public when breastfeeding ${ }^{29}$. Again, perhaps a gradual integration is needed, using techniques to both educate and raise publicity of the benefits of longer - term breastfeeding over time. Alongside inclusion of pictures of older infants being breastfed and a wider acceptance amongst health professionals, greater publication of older infants in books, television programmes and advertising was suggested. Essentially, women wanted longer - term breastfeeding to be more common encountered and seen by a wider section of the public so that over time it became another normal event to see.

In conclusion mothers wanted to feel that their decision to follow a biologically normal act, which is supported by Public Health policy, could also be accepted as normal by their family, Health Professionals and Society as a whole. Making longer-term breastfeeding more visible, re-educating those around the mother and removing the stigma and taboo surrounding feeding an older infant were considered important steps along this journey. 


\section{References}

${ }^{1}$ Ip S, Chung M, Raman G, et al. Breastfeeding and maternal and infant health outcomes in developed countries. 2007;

http://www.ahrq.gov/downloads/pub/evidence/pdf/brfout/brfout.pdf (Accessed 3 February 2011).

${ }^{2}$ Horta BL, Bahl R, Martines JC, Victora CG. Evidence on the long-term effects of breastfeeding: Systematic reviews and meta-analyses. Geneva: World Health Organisation; 2007.

${ }^{3}$ Global Strategy for Infant and Young Child Feeding. World Health Organisation, Geneva, 2002

${ }^{4}$ Department of Health, Breastfeeding. Department of Health webpage, http://www.dh.gov.uk/en/Publichealth/Nutrition/Nutritionpregnancyearlyyears/DH_127625 (Accessed 10 May 2012).

${ }^{5}$ Bolling K, Grant C, Hamlyn, B, Thornton, A. Infant Feeding Survey 2005. The NHS Information Centre for Health and Social Care, 2007; http://www.ic.nhs.uk/pubs/ifs2005 (Accessed 23 September 2011).

${ }^{6}$ Renfrew MJ, Dyson, L, Wallace LM, D'Souza L, McCormick F, Spiby H. Breastfeeding for longer: what works? The Journal of the Royal Society for the Promotion of Health. 2005;125 (2):63-64; 
${ }^{7}$ Pechlivani F, Vassilakou T, Sarafidou J, et al. Prevalence and determinants of exclusive breastfeeding during hospital stay in the area of Athens, Greece. Acta Paediatr 2005;95:928934

${ }^{8}$ Taveras EM, Li R, Grummer-Strawn L, et al. Opinions and practices of clinicians associated with continuation of exclusive breastfeeding. Pediatrics 2004;113:284-290.

${ }^{9}$ Brown A, Lee M. An Exploration of the Attitudes and Experiences of Mothers in the United Kingdom Who Chose to Breastfeed Exclusively for 6 Months Postpartum. Breastfeeding Medicine DOI: $10.1089 / \mathrm{bfm} .2010 .0097$

${ }^{10}$ Gulick EE. The effects of breast-feeding on toddler health. Pediatr Nurs 1986; 12(1):51-54

${ }^{11}$ Lee SY, Kim MT, Kim SW, Song MS, Yoon SJ. Effect of lifetime lactation on breast cancer risk: A Korean Women's Cohort Study. Int J Cancer. 2003 Jun 20;105(3):390-3.

${ }^{12}$ Dettwyler KA. 'A time to wean: The hominid blueprint for the natural age of weaning in modern human populations'. In Stuart-Macadam P, Dettwyler KA, eds. Breastfeeding: Biocultural perspectives. New York: Aldine de Grutyer; 1995:39-73.

${ }^{13}$ Bolling K, Grant C, Hamlyn, B, Thornton, A. Infant Feeding Survey 2005. The NHS Information Centre for Health and Social Care, 2007; http://www.ic.nhs.uk/pubs/ifs2005 (Accessed 23 September 2011).

${ }^{14}$ Scott J, Mostyn T. Women's experiences of breastfeeding in a bottle feeding culture. Journal of Human Lactation, 2003;19:270-277 
${ }^{15}$ Faircloth C. 'It feels right in my heart': affective accountability in narratives of attachment. The Sociological Review; 2001:59(2):283-302.

${ }^{16}$ Dowling S. Women's experiences of long-term breastfeeding. The Practising Midwife; 2009;12(10): 22-25

${ }^{17}$ Dowling S. Online asynchronous and face-to-face interviewing: Comparing methods for exploring women's experiences of breastfeeding long-term. In: Salmons J, ed. Cases in online interview research. Thousand Oaks/London/New Delhi: Sage Publications; 2011: 277296

${ }^{18}$ Asselin ME. Insider research: Issues to consider when doing qualitative research in your own setting. Journal for Nurses in Staff Development; 2003:19(2):99-103.

${ }^{19}$ Dowling S. Inside information: researching long-term breastfeeding. The Practising Midwife; 2009:12(11): 22-26.

${ }^{20}$ Braun V, Clarke V. Using thematic analysis in psychology. Qualitative Research in Psychology; 2006:3:77-101.

${ }^{21}$ Sandelowski M. What's in a name? Qualitative description revisited. Research in Nursing and Health; $2010: 33$ (1):77-84.

${ }^{22}$ Bernard HR. Research methods in anthropology: Qualitative and quantitative approaches. Walnut Creek, CA: AltaMira; 1995.

${ }^{23}$ Creswell JW. Qualitative inquiry and research design: Choosing among five approaches, $2^{\text {nd }}$ ed. Thousand Oaks/London/New Delhi: Sage Publications; 2007.

${ }^{24}$ Lincoln Y, Guba EG. Naturalistic Inquiry. Sage Publications, Newbury Park, CA; 1985. 
${ }^{25}$ Rempel LA. Factors influencing the breastfeeding decisions of long-term breastfeeders. Journal of Human Lactation; 2004:20(3):306-18.

${ }^{26}$ Gribble KD. 'As good as chocolate' and 'better than ice-cream': How toddler, and older, breastfeeders experience breastfeeding. Early Child Development and Care; 2009:10671082. DOI: $10.1080 / 03004430701764176$

${ }^{27}$ Gribble KD. Long-term breastfeeding; changing attitudes and overcoming challenges. Breastfeeding Review; 2008:16(1):5-15.

${ }^{28}$ Department of Health Birth to Five (2009 edition). Department of Health website; 2009. http://www.dh.gov.uk/en/Publicationsandstatistics/Publications/PublicationsPolicyAndGuida $\underline{\text { nce/DH } 107303}$ (Accessed 10 May 2012).

${ }^{29}$ Brown A, Raynor P, Lee M. Young mothers who do breastfeed: The importance of a supportive peer network. Midwifery 2011; 27 (1): 53 - 59. 